\title{
Electroluminescence from an Electrostatically Doped Carbon Nanotube Field-Effect Transistor
}

\author{
M. A. Hughes ${ }^{1, *}$, Y. Ohno ${ }^{2}$, and T. Mizutani2 \\ ${ }^{1}$ Department of Electronic Engineering, Advanced Technology Institute, University of Surrey, \\ Guildford, GU2 7XH, United Kingdom \\ ${ }^{2}$ Department of Quantum Engineering, Nagoya University, Furo-Cho, Nagoya, 464-8603, Japan
}

\begin{abstract}
We report electroluminescence (EL) from a carbon nanotube field-effect transistor with split-gates. $E L$ is generated by the electrostatic doping technique. Six EL bands could be observed, with the strongest band peaking between 0.867 and $0.850 \mathrm{eV}$ with a full width at half maximum (FWHM) of 64 to $120 \mathrm{meV}$, depending on the bias conditions. From the EL peak position we estimate a CNT diameter of $\sim 1.05 \mathrm{~nm}$. We also estimate the power and quantum efficiency of the EL to be around $1 \times 10^{-6}$ and $1 \times 10^{-5}$ respectively. With a fixed drain voltage, increasing the opposite split gate bias caused the EL to increase monotonically from zero, indicating an ambipolar emission mechanism. With a fixed opposite split gate bias the dependence of EL on drain voltage displays a threshold. Drain current is significantly higher when using equal, rather than opposite split gate biases, which we attribute to the trapping of carries by band bending when using opposite split gate bias.
\end{abstract}

Keywords:

Carbon nanotubes (CNTs) are one-dimensional materials with exceptional electronic properties. Their charge carrier mobility can be as high as $79000 \mathrm{~cm}^{2} / \mathrm{Vs},{ }^{1}$ and their current carrying capacity can exceed $1 \times 10^{9} \mathrm{~A} / \mathrm{cm}^{2}{ }^{2}$ Electroluminescence (EL) from CNTs may have applications in nanoscale lasers optical communications. ${ }^{5}$ EL can be obtained from CNT based field-effect transistors (FETs) using various device configurations and mechanisms. The ambipolar mechanism involves the recombination of electrons and holes and requires the simultaneous injection of both these carriers into the CNT. This can be achieved by using asymmetric contacts, where the source and drain contacts are different metals with workfunctions that promote either hole or electron injection. ${ }^{6}$ The electrostatic doping technique utilises split-gates that modify the band profile of CNTs to facilitate EL. ${ }^{3}$ This typically involves the application of a positive bias to one gate and a negative bias to the other gate to dope adjacent section of the nanotube $n$ and $p$ type, respectively, allowing the simultaneous injection of electrons and holes which recombine in an intrinsic region between the spit gates. The unipolar mechanism involves one type of carrier, and EL can occur near trapped charges in the substrate or gate insulator, defects, CNT contacts, or any other inhomogeneities that produce a local electric field capable of accelerating carriers above

\footnotetext{
*Author to whom correspondence should be addressed
}

a threshold energy at which electronic excitation of the CNT across the bandgap can take place. ${ }^{7}$ In this work we report EL from a CNT FET with split-gate geometry, and show that the EL mechanism is ambipolar. The width and peak position of the EL can be varied by switching the polarity of the drain or gate biases.

Devices were fabricated by growing single walled carbon nanotubes (SWNTs) from $0.1 \mathrm{~nm}$ of Co catalyst, patterned by photolithography, on a $\mathrm{SiO}_{2} / \mathrm{Si}$ wafer by ethanol chemical vapour deposition (CVD) at $800^{\circ} \mathrm{C}$. The catalyst was first activated in air at $800{ }^{\circ} \mathrm{C}$. This method produces well separated SWNTs with diameters of 0.5 to $2 \mathrm{~nm}^{8-11}$ Electron beam (EB) lithography was used to pattern source and drain contacts, which were deposited by EB evaporation of $30 \mathrm{~nm}$ of Ti. A gate insulator was formed by atomic layer deposition (ALD) of $40 \mathrm{~nm}$ of $\mathrm{Al}_{2} \mathrm{O}_{3}$ at $300{ }^{\circ} \mathrm{C}$. The split gates (gates 1 and 2) were fabricated by EB lithography and deposition of $\mathrm{Ti} / \mathrm{Au}$. The device structure is illustrated in Figure $1(\mathrm{a}) . I_{d}-V_{\mathrm{ds}}$ scans were obtained using an Agilent B1500 SCS with a minimum current detection limit in our test setup of around $100 \mathrm{fA}$. SEM images were taken using a Zeiss Evo50 at $2 \mathrm{kV}$ using in-line lenses. The contrast mechanism is explained elsewhere. ${ }^{12}$ The SEM image of the device is shown in Figure 1(b), around ten CNTs were observed bridging the channel. EL measurements were taken in air by collecting the EL with a $50 \times$ objective with a focal length of $\sim 1 \mathrm{~cm}$, the EL was then dispersed by a monochromator 
(a)

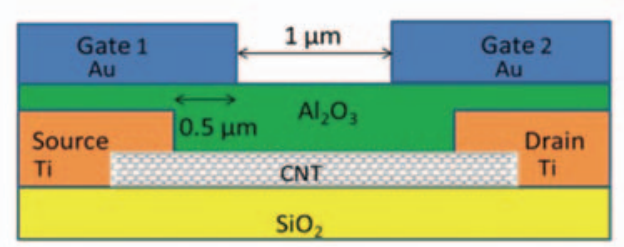

(b)

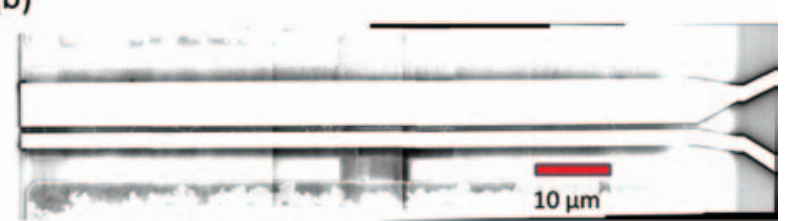

Fig. 1. Schematic cross-section of the device structure (a). SEM image of the device, $\sim$ ten CNTs were observed bridging the channel (b).

and detected using a liquid nitrogen cooled InGaAs detector array. All spectral measurements were corrected for the wavelength dependent response of the measurement system by calculating a correction spectrum $(C(\lambda))$ with: $C(\lambda)=I_{\text {cal }}(\lambda) / I_{\text {meas }}(\lambda)$. Where $I_{\text {meas }}(\lambda)$ is the luminescence spectrum of an Ushio calibrated white light source measured by the detection system and $I_{\text {cal }}(\lambda)$ is the luminescence spectrum of the calibrated white light source supplied by the manufacturer. EL quantum efficiency $\left(\eta_{\mathrm{EL}}\right)$ was estimated by calculating photons per second out and dividing by electrons per second in. Electrons in rate can be calculated from $I_{d} / e$, where $e$ is the electronic charge. To calculate photons out rate we coupled a Ti:sapphire laser at $800 \mathrm{~nm}$ with power $\left(P_{\text {las }}\right)$ and photon energy $\left(Q_{p}\right)$ into the EL measurement system and measured the photoluminescence spectrum $\left(I_{\mathrm{PL}}(\lambda)\right)$ of a bismuth doped glass with known quantum efficiency $\left(\eta_{P L}\right){ }^{13,14}$ The number of photons in the EL spectrum $\left(I_{\mathrm{EL}}(\lambda)\right)$ is proportional to $\int \lambda I_{\mathrm{EL}}(\lambda) C(\lambda) d \lambda$. We multiplied by $\lambda$ to correct for photon energy, since a higher photon flux is required at longer wavelengths to produce the same irradiance per unit area than at shorter wavelengths. Similarly, the number of photons in $I_{\mathrm{PL}}(\lambda)$ is proportional to $\int \lambda I_{\mathrm{PL}}(\lambda) C(\lambda) d \lambda$. Their ratio, multiplied by the actual number of photons in $I_{\mathrm{PL}}(\lambda)=P_{\text {las }} \times \eta_{\mathrm{PL} /} Q_{p}$ gives the photons out rate. Hence $\eta_{\mathrm{EL}}$ can be calculated from Eq. (1)

$$
\frac{I_{d} P_{\mathrm{las}} \eta_{\mathrm{PL}} \int \lambda I_{\mathrm{EL}}(\lambda) C_{\lambda} d \lambda}{e Q_{p} \int \lambda I_{\mathrm{PL}}(\lambda) C_{\lambda} d \lambda}
$$

Figure 2(a) shows the $I_{d}-\mathrm{V}_{\mathrm{ds}}$ characteristics of the device. The device should be in forward bias when gate 2 has an opposite polarity to $V_{\mathrm{ds}}$, a band diagram representing this situation is shown in Figure 2(c). The device should be in reverse bias when gate 2 has the same polarity as $V_{\mathrm{ds}}$. The reason for this is that total deflection of the valance and conduction bands is at a maximum when gate 2 has the opposite polarity to $V_{\mathrm{ds}}$ and at a minimum when gate 2 has the same polarity as $V_{\mathrm{ds}}$. The inset of Figure 2(a) shows a close-up of the $I_{d}-V_{\mathrm{ds}}$ characteristics using opposite split gate biases. They are largely symmetric and in both forward and reverse bias configuration and there is a respective increase or decrease in $I_{d}$ of $\sim 5 \%$. Using opposite split gate bias should produce a rectifying $p-n$ junction. The lack of rectification in the $I_{d}-V_{\mathrm{ds}}$ characteristics can be explained by the presence of metallic CNTs that would mask the rectifying

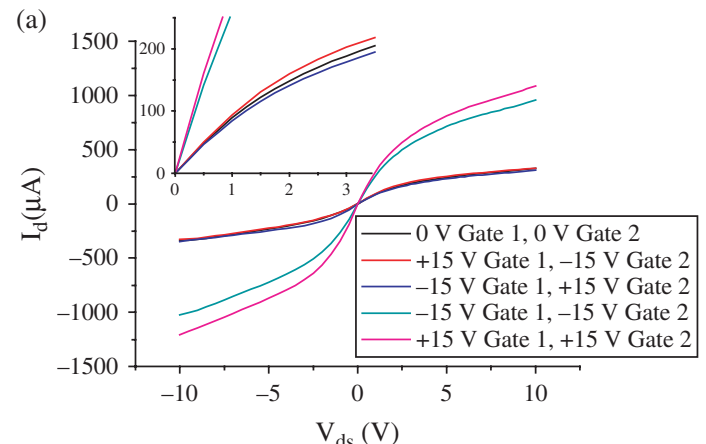

(c)

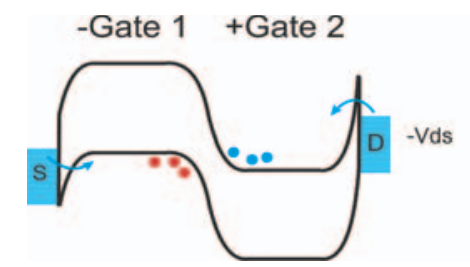

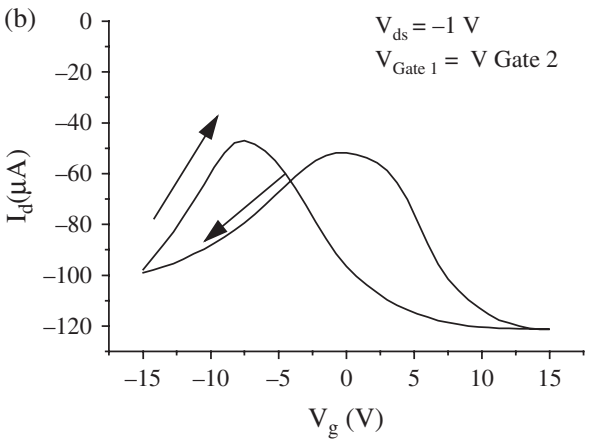

(d)

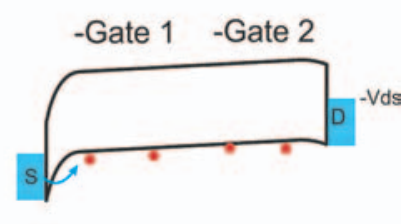

Fig. 2. $I_{d}-V_{\mathrm{ds}}$ characteristics of the device at various split gate biases (a). Transfer characteristic with equal split gate bias and $V_{\mathrm{ds}}=-1 \mathrm{~V}$ (b). Band diagram representing the device in forward bias configuration with gate $1,2=-15,+15 \mathrm{~V}$, illustrating how carriers can be trapped by band bending in this configuration (c). Band diagram representing the symmetric device with equal split gate bias, illustrating how carriers can flow freely in this configuration (d). Blue and red circles circle represent electrons and holes, respectively. Blue arrows represent tunnelling transport. 
behaviour of the semiconducting CNTs. This rectifying behaviour is only apparent as small respective increases and decreases in $I_{d}$ in the forward and reverse bias configurations. Figure 2(b) shows the transfer characteristics with equal gate bias. The device shows ambipolar characteristics, indicating the ability to inject holes and electrons. The on-off ratio is $\sim 2$ and we suggest that the off-state current is caused either by the presence of some metallic CNTs, or the superposition of transfer characteristics of multiple CNTs with different off-state gate potentials. The hysteresis in the transfer characteristics indicates the presence of trapped charges (holes or electrons) in the substrate and/or gate insulator. ${ }^{15,16}$ Figure 2(a) shows that $I_{d}$ is significantly larger when using equal, rather than opposite split gate biases. In order to explain this we propose that when opposite gate polarities are applied to the device, carriers can become trapped by band bending, as illustrated in Figure 2(c). Whereas when equal gate bias of the same polarity is applied to the device carriers can move freely, as illustrated in Figure 2(d). A similar effect has been illustrated, but not explained, in comparable device structures. ${ }^{17}$ Although it is possible that our device contains some metallic CNTs, these should not be modified by the gate bias, therefore our band diagrams illustrating the effect of the gate bias represent semiconducting CNTs.

Figure 3 shows contour plots of EL spectra as a function of drain bias for gate $1,2=-15,+15 \mathrm{~V}$ (b) and gate $1,2=+15 \mathrm{~V},-15 \mathrm{~V}(\mathrm{c})$, constituting four forward or reverse configurations. There is a strong EL peak at $\sim 0.86 \mathrm{eV}$. For a CNT emitting at this energy, embedded in $\mathrm{Al}_{2} \mathrm{O}_{3} / \mathrm{SiO}_{2}\left(\varepsilon_{\text {eff }} \approx 5.7\right)$, we estimate an exciton binding energy of $0.12 \mathrm{eV},{ }^{18}$ giving an estimated CNT diameter of $\sim 1.05 \mathrm{~nm} .{ }^{19,20}$ There are also several other weaker EL bands extending up to $1.2 \mathrm{eV}$. The weakness of the higher energy bands could be due to the difficulty of electrically exciting wide bandgap SWNTs. ${ }^{21}$ The results in Figure 5 indicate that EL produced with a split gate bias is generated by an ambipolar mechanism, i.e., from the recombination of holes and electrons. The EL intensity can therefore be seen as a proxy for the recombination current. The strength of the EL for the various forward and reverse bias configurations can be represented as three levels: forward bias in Figure 3(b) is strong, reverse bias in Figure 3(c) is weak, and reverse bias in Figure 3(b) and forward bias in Figure 3(c) are

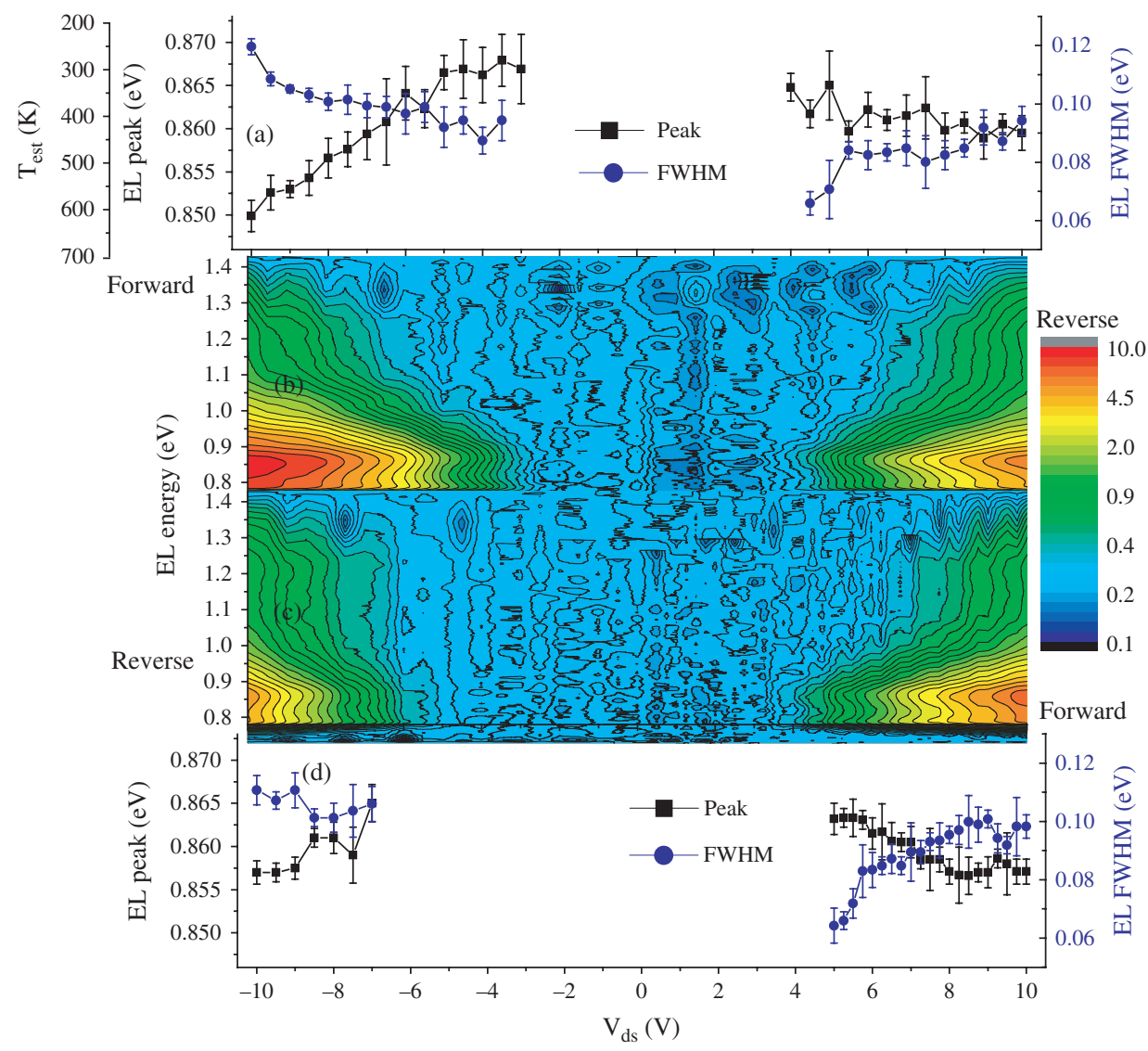

Fig. 3. Contour plot of EL spectra as a function of drain bias for gate $1,2=-15 \mathrm{~V},+15 \mathrm{~V}$ (b) and gate $1,2=+15 \mathrm{~V},-15 \mathrm{~V}$, (c) each forward or reverse bias configuration is marked, the intensity is in a $\log$ scale. The peak position and FWHM of the main EL peak, derived from deconvolution of the EL spectra into Gaussians, are shown for gate $1,2=-15 \mathrm{~V},+15 \mathrm{~V}$ (a) and gate $1,2=+15 \mathrm{~V},-15 \mathrm{~V}$ (d). The lattice temperature $\left(T_{\text {est }}\right)$, estimated from the shift of the EL peak position, is shown on a second $y$-axis in (a). 
both intermediate. This asymmetry of the EL intensity for the different forward and reverse bias configurations can be explained by the high drain bias, which implies that the total deflection of the conduction and valence bands by the gate is proportional to the sum of the gate and drain biases at the drain, but only the gate potential at the source. This results in electron injection into the CNT by tunnelling being easiest for the forward bias configuration in Figure 3(b), most difficult for the reverse bias configuration in Figure 3(c), and intermediate for configurations of reverse bias in Figure 3(b) and forward bias in Figure (c).

Individual EL spectra, deconvoluted into Gaussian components, from the device in forward bias with $V_{\mathrm{ds}}=-10 \mathrm{~V}$ and $-4 \mathrm{~V}$ are provided in Figures $4(\mathrm{a})$ and (b), respectively. The FWHM increases from $87 \mathrm{meV}$ to $120 \mathrm{meV}$ as $V_{\mathrm{ds}}$ is increased from -4 to $-10 \mathrm{~V}$. Figures 3(a) and (d) show the EL FWHM as a function of $V_{\mathrm{ds}}$. The minimum EL FWHM, at $64 \mathrm{meV}$, is lowest for positive $V_{\mathrm{ds}}$ with both gate configurations. We can therefore vary the EL FWHM by reversing both split gate and drain polarities. We expect changes in the EL peak position and FWHM with increasing $V_{\mathrm{ds}}$ to be related to Joule heating which should cause a red shift in the EL peak position ${ }^{22}$ and broadening of the EL peak due to increased thermal and lifetime broadening effects. ${ }^{23}$ The bandgap $\left(E_{g}\right)$ for most SWNTs decreases linearly with temperature with a slope of around $-5 \times 10^{-5} \mathrm{eV} /{ }^{\circ} \mathrm{C},{ }^{24}$ enabling the shift in EL peak position to be used to estimate lattice temperature $\left(T_{\text {est }}\right)$. As $V_{\mathrm{ds}}$ increases from 3 to $5 \mathrm{~V}$ in the forward bias region of Figure 3(a), the main EL peak and FWHM remain relatively unchanged, indicating that no significant heating of the CNT occurs in this voltage range. For $V_{\mathrm{ds}}$ biases above $5 \mathrm{~V}$ the main EL peak shifts to lower energy and broadens. We assume the lattice temperature is around $300 \mathrm{~K}$ in the 3 to $5 \mathrm{~V} V_{\mathrm{ds}}$ range where the EL peak is $\sim 0.867 \mathrm{eV}$. $T_{\text {est }}$ was then calculated from the peak shift $(\Delta \mathrm{EL})$ by $T_{\text {est }}=\left(\Delta E L / 5 \times 10^{-5}\right)+300$. As shown in Figure 3(a), the lattice temperature reached an estimated $650 \mathrm{~K}$ at $V_{\mathrm{ds}}=-10 \mathrm{~V}$.

At $V_{\mathrm{ds}}=-10 \mathrm{~V}$, and gate $1,2=-15 \mathrm{~V},+15 \mathrm{~V}$, an excellent fit of the EL spectrum was obtained using a sum of 5 Gaussian emitters, see Figure 4(a). We also identified a low energy band that lies mostly outside the detector range, but can be seen as a deviation from the Gaussian fit. This indicates a total of six EL emitters. We suggest that each band originates from an individual CNT which is in good agreement with the SEM observation of $\sim$ ten CNTs bridging the channel. The unaccounted CNTs may be metallic or poorly contacted. We estimate the maximum power and quantum efficiency of the EL to be around $1 \times$ $10^{-6}$ and $1 \times 10^{-5}$, respectively. This compares to quantum efficiencies of $1 \times 10^{-4}$ in single CNT split gate diodes $10^{-9}$ for electrolyte gated CNT FETs, ${ }^{5}$ and $1 \times 10^{-6}$ for CNT network transistors. ${ }^{25}$ The FWHM of the main EL peak varied between 64 and $120 \mathrm{meV}$ over all bias configurations. Typically, an EL FWHM $>150 \mathrm{meV}$ indicates hot carriers are involved in EL process, ${ }^{26}$ hence the observed EL FWHM suggests that hot carriers are not involved.

Figure 5 shows the integrated EL intensity as a function of increasing opposite polarity split gate bias, with $V_{\mathrm{ds}}=$ $-5 \mathrm{~V}$, putting the device into an increasing forward bias configuration. There is a small amount of EL at zero gate bias which should be due to a unipolar mechanism. The EL intensity then reduces to zero with increasing opposite polarity split gate bias before increasing approximately linearly with split gate bias. In Figure $5, I_{d}$ increases by a relatively small amount from $215 \mu \mathrm{A}$ to $255 \mu \mathrm{A}$ when the split gate bias is increased from zero to $\pm 17 \mathrm{~V}$. This indicates that there is no contribution of blackbody emission to the EL spectra because blackbody emission should depend only on $I_{d}$ and not be affected by the gate bias. At zero gate bias we propose that $I_{d}$ consists mostly of majority carriers or current through metallic CNTs, since we observe little EL. The increase in $I_{d}$, caused by the
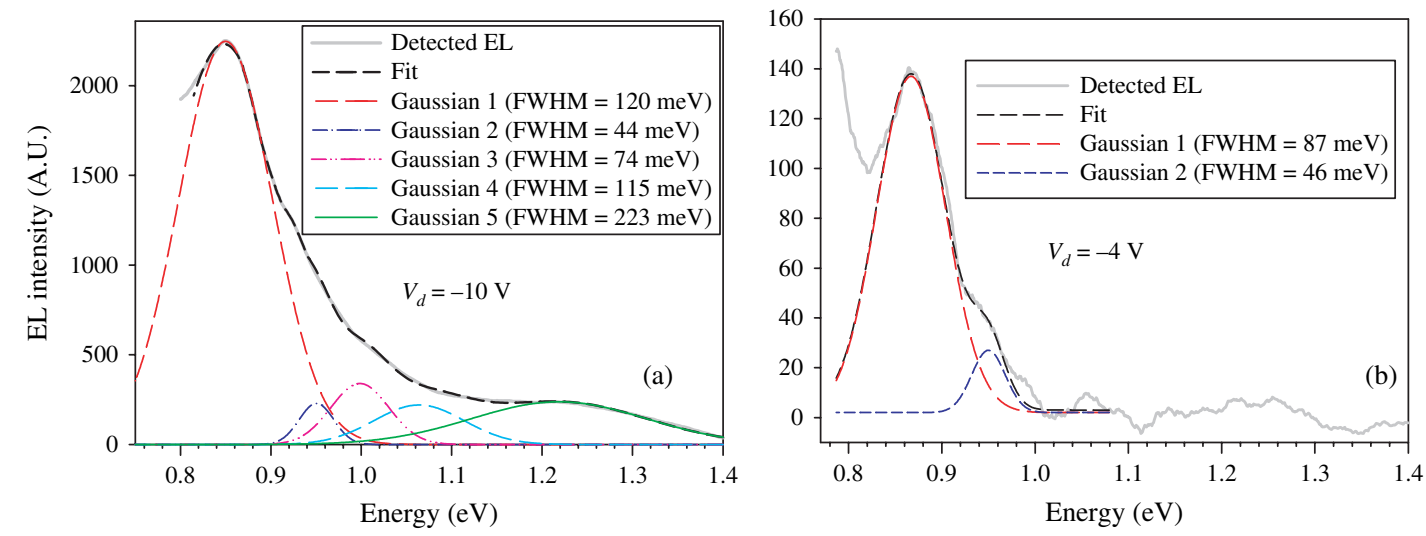

Fig. 4. EL spectra from $V_{\mathrm{ds}}=-10 \mathrm{~V}$ (a) and $-4 \mathrm{~V}$ (b), deconvoluted into Gaussians, with gate $1=-15 \mathrm{~V}$, gate $2=+15 \mathrm{~V}$. We fitted the minimum number of Gaussians required to give a coefficient of determination $\left(R^{2}\right)>0.995$. 


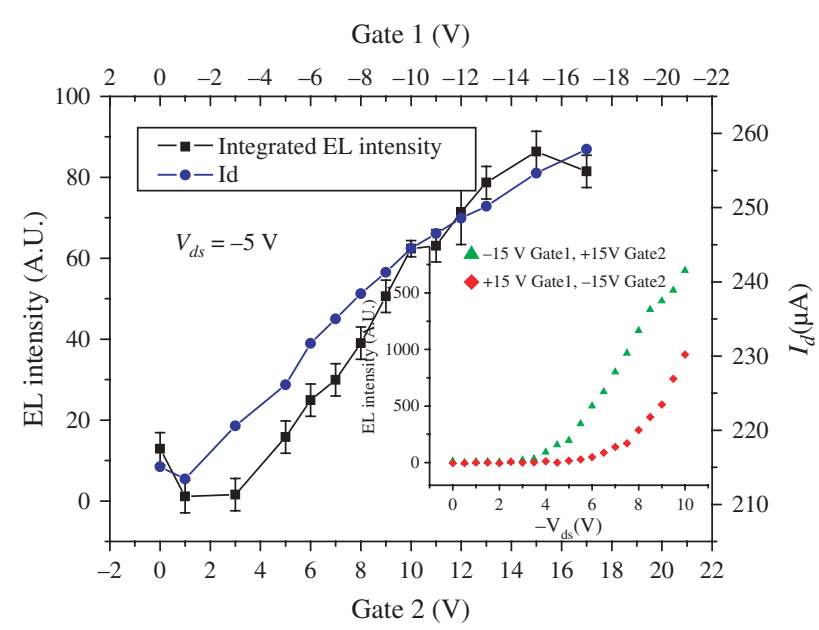

Fig. 5. Integrated EL intensity and drain current as a function of split gate bias, with a drain bias fixed at $-5 \mathrm{~V}$. Inset shows the integrated EL intensity as a function of drain bias with split gate biases of gate $1,2=-15,+15 \mathrm{~V}$ (corresponding to forward bias) and gate 1 , $2=+15 \mathrm{~V},-15 \mathrm{~V}$ (corresponding to reverse bias). When integrated EL intensity is plotted as a function of $I_{d}$, the dependence is broadly similar.

split gate bias, must be due to the injection of minority carriers, which can produce EL by an ambipolar mechanism. This is because the overall increase in $I_{d}$ is so small that the EL shouldn't be generated by a unipolar mechanism. The inset of Figure 5 shows the integrated EL intensity as a function of $V_{\mathrm{ds}}$ for split gate biases of gate $1,2=-15,+15 \mathrm{~V}$ (forward bias) and gate $1,2=+15$, $-15 \mathrm{~V}$ (reverse bias). It shows that EL is stronger in forward bias than reverse bias for the same $V_{\mathrm{ds}}$. Forward bias should promote $\mathrm{e}-\mathrm{h}$ pair recombination and therefore the increase in EL should be due to $\mathrm{e}-\mathrm{h}$ pair recombination. EL in reverse bias should originate from a unipolar mechanism. The general trend of EL intensity is similar when it is plotted as a function of input power $\left(I_{d} V_{\mathrm{ds}}\right)$ or $I_{d}$, with each plot showing a linear relationship above a threshold. A linear dependence of EL intensity on $I_{d}$ has also been reported for ambipolar CNT FETs. ${ }^{21}$ It has been reported that a thresholdless dependence of EL power on electrical power indicates e-h pair recombination by an ambipolar mechanism. ${ }^{17}$ The presence of a threshold for EL indicates a unipolar mechanism occurs, where electrons or holes are accelerated to energies sufficient to generate excitons by impact excitation. ${ }^{7} 17$ However, the gate dependence of EL in Figure 5 indicates the presence of a $p-n$ junction and an ambipolar mechanism. We propose that this apparent contradiction occurs because there is a larger Schottky barrier height for holes than for electrons This would result in the EL intensity being dependent on the hole current, which we would expect to have a threshold-like dependence on $V_{\mathrm{ds}}$.

In summary, the $I_{d}-V_{\mathrm{ds}}$ characteristics show $I_{d}$ is larger when using equal, rather than opposite split gate biases, which we attribute to the trapping of carries by band bending when using opposite split gate bias. $I_{d}$ changes by $\sim 5 \%$ in the expected direction when operated in forward or reverse bias configurations. Ambipolar transfer characteristics were observed with an on/off ratio of $\sim 2$. Six EL bands could be observed with the strongest band peaking at $0.86 \mathrm{eV}$. The EL FWHM varied from 64 to $120 \mathrm{meV}$, depending on the bias conditions. These FWHMs indicate that hot carriers are not involved in the EL process. EL FWHM can be varied by reversing both split gate and drain polarities. We estimate the power and quantum efficiency of the EL to be around $1 \times 10^{-6}$ and $1 \times 10^{-5}$ respectively. With a fixed $V_{\mathrm{ds}}$, increasing the opposite split gate bias caused the EL to increase monotonically from zero, indicating an ambipolar emission mechanism. With a fixed opposite split gate bias the dependence of EL on $V_{\mathrm{ds}}, I_{d} V_{\mathrm{ds}}$ or $I_{d}$ displays a threshold, indicating a unipolar emission mechanism.

\section{References and Notes}

1. T. Dürkop, S. A. Getty, E. Cobas, and M. S. Fuhrer, Nano Lett. 4, 35 (2004).

2. Z. Yao, C. L. Kane, and C. Dekker, Phys. Rev. Lett. 84, 2941 (2000).

3. T. Mueller, M. Kinoshita, M. Steiner, V. Perebeinos, A. A. Bol, D. B. Farmer, and P. Avouris, Nat. Nanotechnol. 5, 27 (2010).

4. A. Högele, C. Galland, M. Winger, and A. Imamoğlu, Phys. Rev. Lett. 100, 217401 (2008).

5. J. Zaumseil, X. N. Ho, J. R. Guest, G. P. Wiederrecht, and J. A. Rogers, ACS Nano 3, 2225 (2009).

6. X. Xie, A. E. Islam, M. A. Wahab, L. Ye, X. Ho, M. A. Alam, and J. A. Rogers, ACS Nano 6, 7981 (2012).

7. P. Avouris, M. Freitag, and V. Perebeinos, Nat. Photonics 2, 341 (2008).

8. N. Fukuoka, Y. Mizutani, S. Naritsuka, T. Maruyama, and S. Iijima, Jpn. J. Appl. Phys. 51, 06FD23 (2012).

9. T. Mizutani, Y. Nosho, and Y. Ohno, J. Phys. Conf. Ser. 109, 012002 (2008).

10. Y. Mizutani, N. Fukuoka, S. Naritsuka, T. Maruyama, and S. Iijima, Diam. Relat. Mat. 26, 78 (2012).

11. S. Noda, H. Sugime, T. Osawa, Y. Tsuji, S. Chiashi, Y. Murakami, and S. Maruyama, Carbon 44, 1414 (2006).

12. M. A. Hughes, K. P. Homewood, R. J. Curry, Y. Ohno, and T. Mizutani, Appl. Phys. Lett. 103, 133508 (2013).

13. M. Hughes, T. Suzuki, and Y. Ohishi, Journal of the Optical Society of America B: Optical Physics 25, 1380 (2008).

14. M. Hughes, T. Suzuki, and Y. Ohishi, J. Non-Cryst. Solids 356, 407 (2010).

15. W. Kim, A. Javey, O. Vermesh, O. Wang, Y. M. Li, and H. J. Dai, Nano Lett. 3, 193 (2003).

16. M. S. Fuhrer, B. M. Kim, T. Dürkop, and T. Brintlinger, Nano Lett. 2, 755 (2002).

17. M. Kinoshita, M. Steiner, M. Engel, J. P. Small, A. A. Green, M. C. Hersam, R. Krupke, E. E. Mendez, and P. Avouris, Opt. Express 18, $25738(\mathbf{2 0 1 0})$

18. V. Perebeinos, J. Tersoff, and P. Avouris, Phys. Rev. Lett. 92, 257402 (2004).

19. S. M. Bachilo, M. S. Strano, C. Kittrell, R. H. Hauge, R. E. Smalley, and R. B. Weisman, Science 298, 2361 (2002). 
20. R. B. Weisman and S. M. Bachilo, Nano Lett. 3, 1235 (2003).

21. N. Hibino, S. Suzuki, H. Wakahara, Y. Kobayashi, T. Sato, and H. Maki, ACS Nano 5, 1215 (2011).

22. L. Xie, H. Farhat, H. Son, J. Zhang, M. S. Dresselhaus, J. Kong, and Z. Liu, Nano Lett. 9, 1747 (2009).

23. D. Mann, Y. K. Kato, A. Kinkhabwala, E. Pop, J. Cao, X. R. Wang, L. Zhang, Q. Wang, J. Guo, and H. J. Dai, Nat. Nanotechnol. 2, 33 (2007).
24. R. B. Capaz, C. D. Spataru, P. Tangney, M. L. Cohen, and S. G. Louie, Phys. Rev. Lett. 94, 036801 (2005).

25. E. Adam, C. M. Aguirre, L. Marty, B. C. St-Antoine, F. Meunier, P. Desjardins, D. Menard, and R. Martel, Nano Lett. 8, 2351 (2008).

26. M. Freitag, V. Perebeinos, J. Chen, A. Stein, J. C. Tsang, J. A. Misewich, R. Martel, and P. Avouris, Nano Lett. 4, 1063 (2004).

Received: $\mathrm{xx}$ xx Xxxx. Accepted: $\mathrm{xx}$ xx Xxxx. 